\title{
Research and Design of Motion Control System for Automated Warehouse
}

\author{
Wang Guangdong ${ }^{1}$, Zhu Wu ${ }^{1}$, Zhang Jiamin ${ }^{2}$ \\ ${ }^{1}$ School of Electronics and Information Engineering, Shanghai University of Electric Power, Shanghai, China \\ ${ }^{2}$ School of Automation Engineering, Shanghai University of Electric Power, Shanghai, China
}

\section{Email address:}

282498575@qq.com (Wang Guangdong),1483075722@qq.com (Zhu Wu),657758015@qq.com (Zhang Jiamin)

\section{To cite this article:}

Wang Guangdong, Zhu Wu, Zhang Jiamin. Research and Design of Motion Control System for Automated Warehouse. Science Discovery. Vol. 5, No. 3, 2017, pp. 229-235. doi: 10.11648/j.sd.20170503.22

Received: March 5, 2017; Accepted: May 13, 2017; Published: May 16, 2017

\begin{abstract}
The purpose of this paper is to improve the performance of flexible welding production line in the automated warehouse stacking machine and auto warehouse operation efficiency, for the first time with a control structure of motion controller SIMOTION+ servo driver + servo motor ", the use of SIMOTION technology can realize accurate advantage of multi axis motion control and logic control of the machine in the same system, to realize the control of the stacking machine stable and accurate operation.
\end{abstract}

Keywords: Automated Warehouse, Stacker, SIMOTION, PLC, Servo Control

\section{自动化立体仓库运动控制系统的研究与设计}

王光东 ${ }^{1}$, 朱武 $^{1}$, 张佳民 $^{2}$

${ }^{1}$ 电子信息工程学院, 上海电力学院, 上海市, 中国

${ }^{2}$ 自动化工程学院, 上海电力学院, 上海市, 中国

邮箱

282498575@qq.com（王光东），1483075722@qq.com（朱武），657758015@qq.com（张佳民）

摘要: 本文主要是为了提高汽车柔性焊接生产线自动化立体仓库中堆垛机的性能及立体仓库的作业效率, 首次采用了 “运动控制器SIMOTION+伺服驱动器+伺服电机” 的控制结构, 利用SIMOTION可在同一个系统中实现精确地多轴运动控 制和机器逻辑控制的技术优势, 实现了对堆垛机稳定、精确的运行控制。

关键字：自动化立体仓库，堆垛机，SIMOTION, PLC, 伺服控制

\section{1. 引言}

目前, 随着工业自动化水平在汽车制造行业的不断提 高, 自动化立体仓库技术被迅速引进到汽车制造行业中, 其对汽车生产线的整体作业效率具有重要影响。堆垛机作 为立体仓库中的核心设备, 具有较高的科技含量, 决定着 自动化立体仓库的技术水平及工作效益。因此, 采用先进
的控制技术，不断改善并提高堆垛机的性能是必然趋势 [1]。

自动化立体仓库是一个庞大且精细的复杂系统, 计算 机调度系统和上位机监控系统是其 “大脑” , 传感器检测 系统与RFID识别系统是其 “眼睛” , 钢结构货架和轨道等 硬件是其 “骨骼”, 各级子系统进行信息交互的通讯网络 是其 “经络” ，堆垛机搬运系统和转台是其 “四肢”，还 
有其它硬件设备共同运转, 完成焊接夹具在汽车柔性生产 线中的精准、快速、高效、自动的出入库作业 [2]。

在电气控制方面, 国内自动化立体仓库普遍采用的是 “PLC+变频器+三相异步交流电机” 的控制结构, 这种结 构基本上可以满足自动化立体仓库对堆垛机快速性、精准 性和稳定性的要求, 但面对当前自动化立体仓库越来越大 的存储空间, 越来越高的堆垛机高度和越来越快的运行速 度等发展形势, 这种控制结构越来越力不从心, 较国外采 用更加先进的伺服控制系统的堆垛机，国内堆垛机在定位 精度和作业效率等主要技术指标上有较大差距 [3]。

本文以某汽车柔性生产线的焊接夹具自动化立体仓 库为研究对象, 并对自动化立体仓库中堆垛机控制系统进 行了深入研究。首先将自动化立体仓库系统分成了计算机 调度系统、上位机监控系统、主站PLC控制系统、子站堆 垛机SIMOTION运动控制系统和设备层系统五个子系统进 行深入分析, 并重点对设备层系统中的堆垛机控制系统进 行了深入研究, 详细阐述了堆垛机控制系统设计方案, 主
要包括电气控制系统方案 (速度控制和定位控制) 和通讯 系统方案。

\section{2. 立体仓库控制系统整体架构}

本课题所研制的自动化立体仓库是一个复杂的运动 系统, 主要由控制系统和底层硬件组成, 其中, 控制系统 首次采用了 “运动控制器SIMOTION + 伺服驱动器 + 伺服电机” 的控制结构, 融合了先进的自动控制技术、高速通讯技术 和现场总线技术等, 实现了各个设备高速、精准的协调运 转, 能实时根据生产线的生产需求自动完成焊接夹具平稳、 高效的出入库作业 $[4]$ 。

自动化立体仓库的总体系统可以分成五个层级的子 系统, 即计算机调度系统、上位机监控系统、主站PLC控 制系统、子站堆垛机SIMOTION运动控制系统和设备层系统, 各层级子系统通过通讯网络进行信息的交互, 其控制系统 结构图如图1所示。

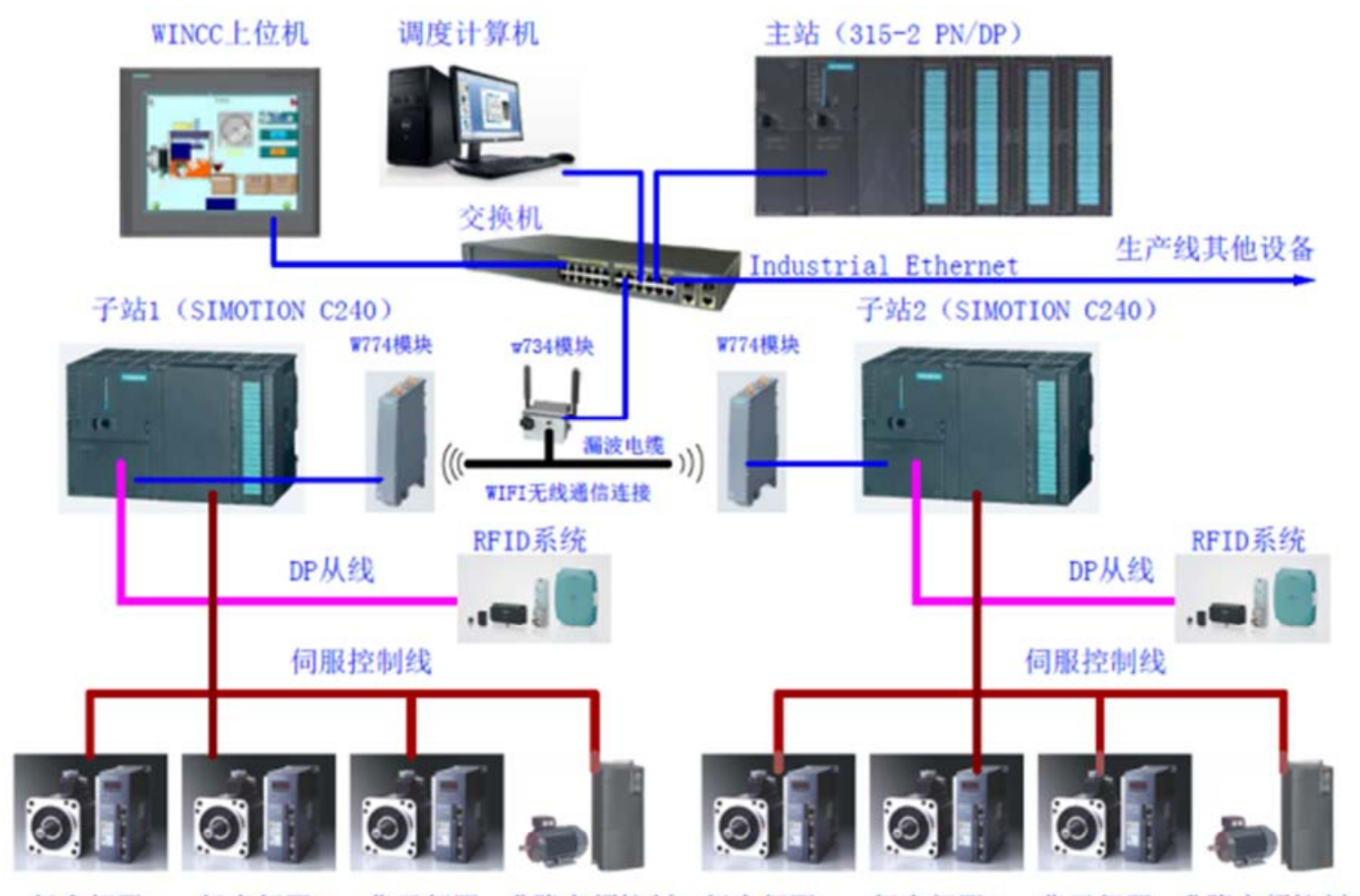

行走何服1 行走何服2 货叉何服升降变频控制 行走何服1 行走伺服2 货叉何服 升降变频控制

图1 立体仓库控制系统结构图。

两台堆垛机作为整个自动化立体仓库的核心设备, 其 采用运动控制器SIMOTION 作为主控设备, 夹具RFID识别 系统、激光测距保护系统、断绳检测保护系统和货位数据 库系统是其辅助系统, 并通过工业以太网与上层系统进行 通信 [5]。本课题所研究的立体仓库控制系统结构拓扑图 如图2所示。

\section{1. 计算机调度系统}

计算机调度系统处于顶层, 采用主流的Windows 7 操 作系统, 可通过网络访问服务器数据库, 负责与生产线的
信息对接, 下发给上位机生产线需要存/取的焊接夹具种 类和每种夹具的数量等信息, 使立体仓库和生产线能够进 行有机地结合、各个设备协调运作。

\section{2. 上位机监控系统}

上位机监控系统 (如图3所示) 处于次顶层, 在解析 调度信息后, 依次发出焊接夹具的出/入库命令, 逐个完 成焊接夹具的存/取, 且发生异常情况时, 可通过手动模 式完成自动化立体仓库的整体自动化作业。 


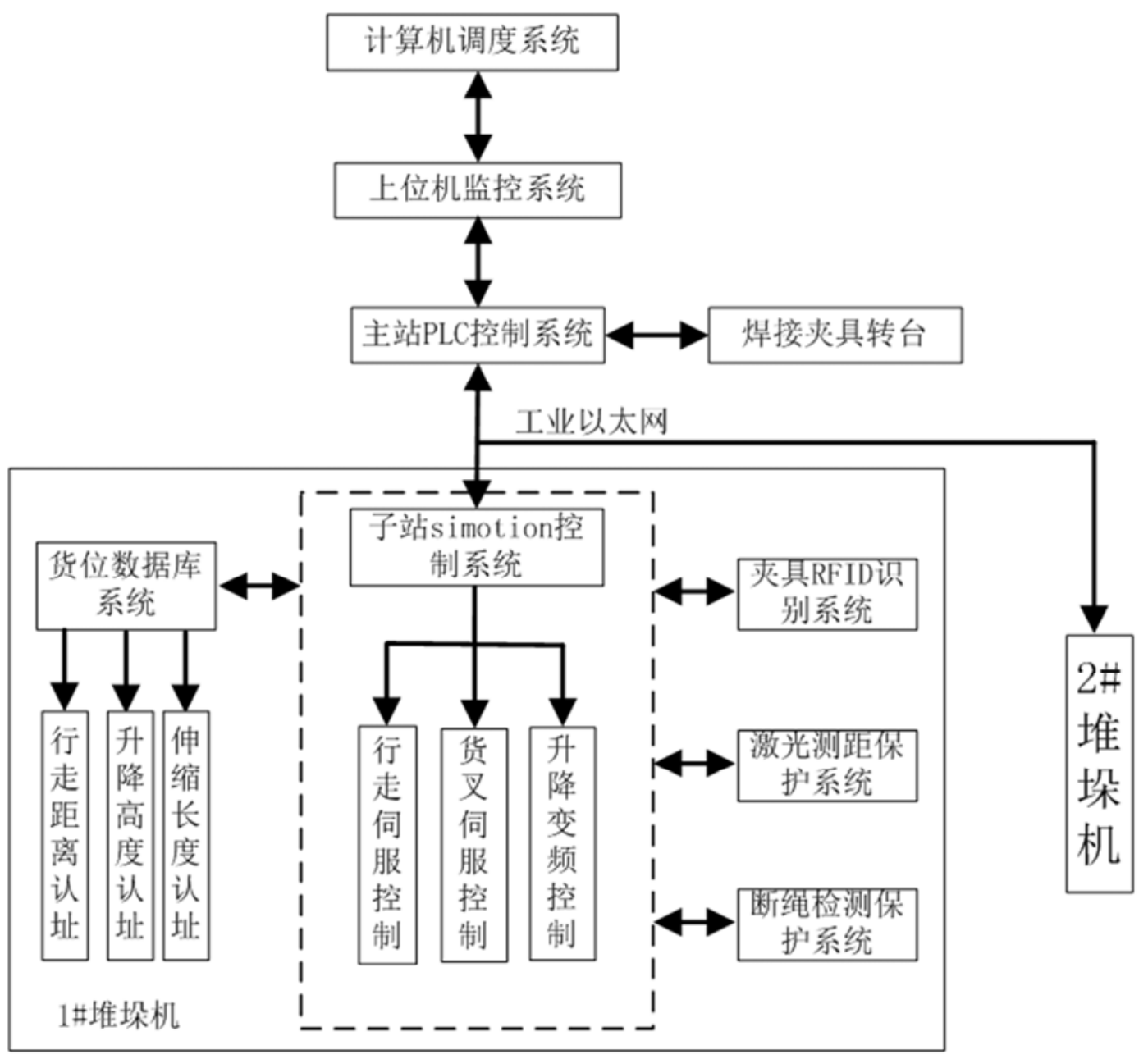

图2 立体仓库控制系统结构拓扑图。

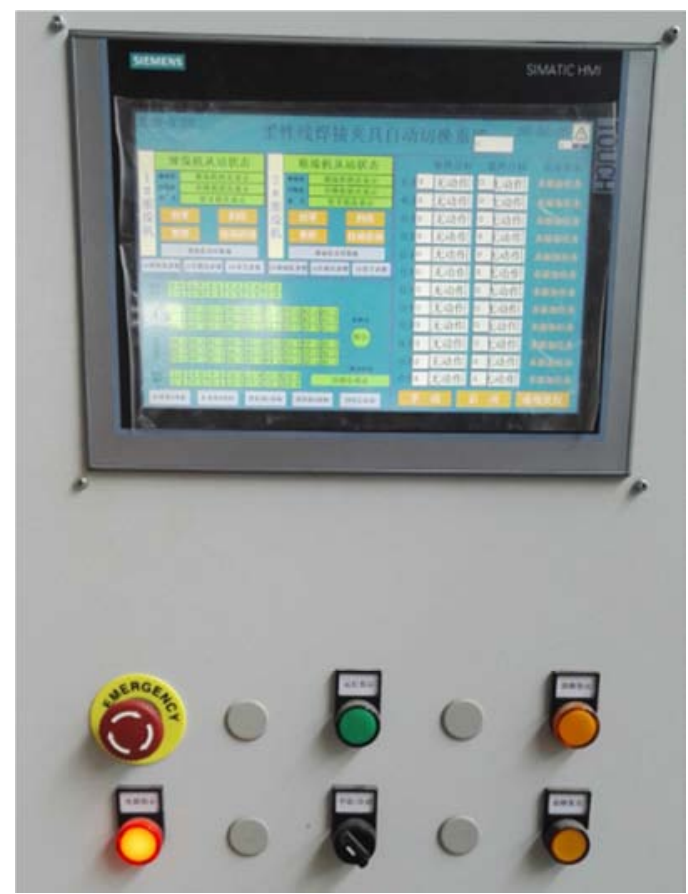

图3 上位机监控系统。

\section{3. 主站PLC控制系统}

主站PLC控制系统 (如图4所示) 处于次底层, 主要工 作是解析上位机下发的作业命令, 规划堆垛机的运动路径 和动作流程，根据两个子站的工作状态下发控制命令，同 时需完成对各个传感器装置在堆垛机自动运行过程中的 数据采集工作。主站电气柜主要安装有PLC及其扩展模块、 触摸屏、交换机和w734通信模块等设备。

\section{4. 子站堆垛机运动控制系统}

两个子站运动控制器SIMOTION是最底层控制系统, 子 站电气柜安装于堆垛机上, 主要负责控制堆垛机水平行走 机构、垂直升降机构和货叉伸缩机构的协调运作，使其按 照主站命令完成焊接夹具的存取, 并且以一定时间间隔向 主站发送当前工作状态数据 [6]。子站电器柜主要装有 simotion运动控制器、伺服驱动器、变频器、w774无线通 信模块等设备, 其电气柜如图5所示。

\section{5. 设备层系统}

设备层系统主要由水平行走机构、垂直升降机构、货 叉伸缩机构、旋转台、立体货架和外围设备等组成。 


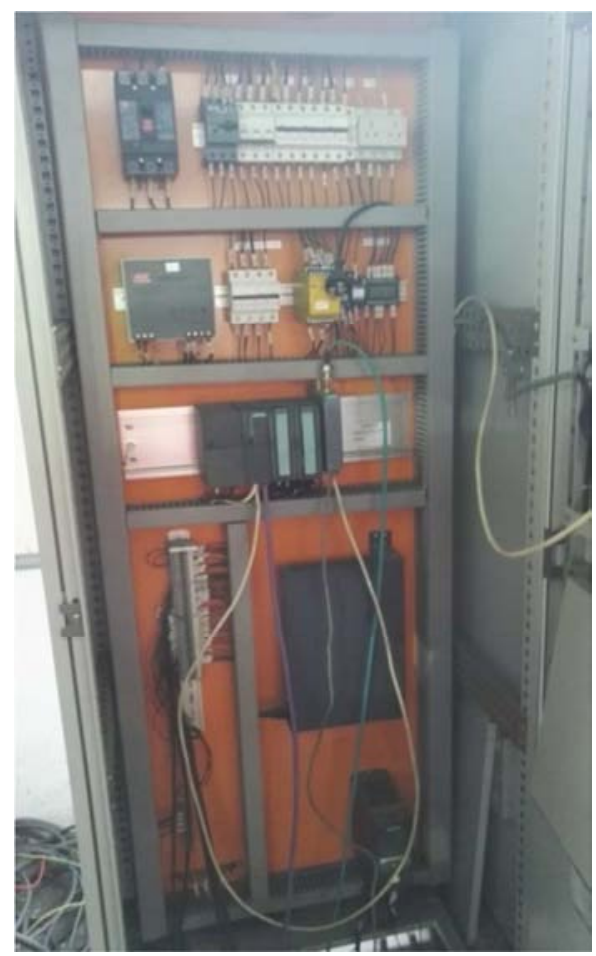

图4 主站PLC电气柜。

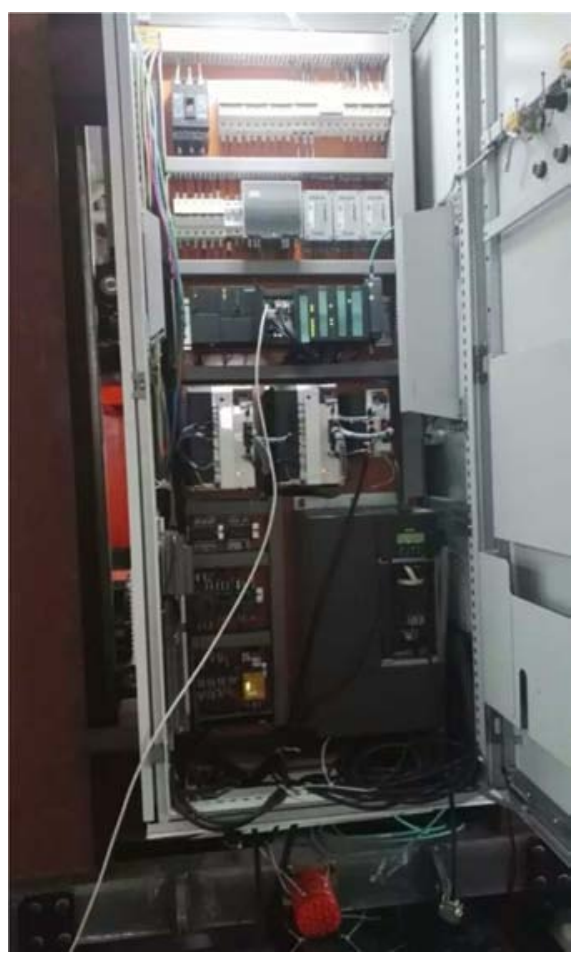

图5 子站SIMOTION电气柜。

\section{3. 堆垛机控制系统方案设计}

堆垛机设计主要包括电气设计部分和机械设计部分, 本文的研究重点是堆垛机的电气控制系统设计。堆垛机的 主要工作是穿梭于立体仓库之中完成焊接夹具的出/入库 作业, 其必须具备水平行走、垂直升降和货叉伸缩三个方
向的运动, 并且具备较好的速度控制和精确的定位功能 [7], 因此, 控制系统的设计是决定堆垛机性能优劣的主 要因素。本课题采用 “运动控制器SIMOTION+伺服驱动器 + 伺服电机” 的电气控制结构, 以必要的硬件电路设计为依 托，控制策略及算法为控制核心，基于标准TCP协议的无 线通信为信息交互平台，实现对堆垛机平稳、精确的自动 化控制。

\section{1. 堆垛机控制系统总体结构}

本课题研究的自动化立体仓库系统包括两台堆垛机, 每台堆垛机的控制系统分为二级结构如图6所示, 控制器 SIMOTION直接控制伺服驱动器与电机, 并读取安装在堆垛 机上面的传感器检测信息, 使堆垛机完成各项动作。

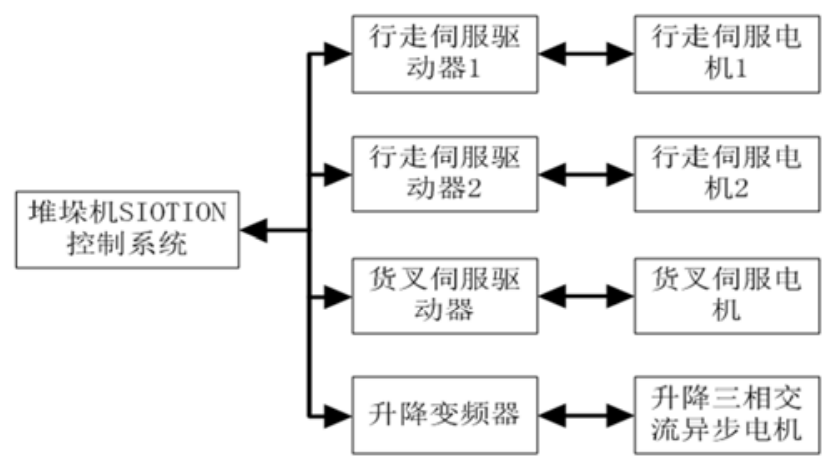

图6 堆垛机控制系统框图。

堆垛机需在巷道上来回移动搬运焊接夹具, 但这样会 为接线带来很多不便，使用无线通讯后堆垛机的控制自成 一体, 信号交互通过无线完成传输, 具体实施办法是将西 门子无线通信模块W734 (发射器) 连接到交换机, 巷道下 铺设有漏波电缆, 而西门子无线通信模块W774 (接收器) 安装于堆垛机上, 这样大大提高了堆垛机的活动范围及灵 活性。

无线通信模块W774上具有两个PN口, 一个连接到控制 器SIMOTION C240上, 另一个连接到激光测距模块上。 SIMOTION的X2接口, 可作为 \pm 10 VDC 模拟量或步进电机接 口输出, 最多可以控制 4 个轴, 用于驱动控制两个行走伺 服轴、一个货叉伺服轴和一个变频升降轴。SIMOTION有四 个编码器接口, 分别是X3、X4、X5和X6, 用来接两个行走 伺服电机的编码器和两个拉线编码器。SIMOTION C240提 供有两个PROFIBUS DP接口, 分别用来接RFID模块和货叉 编码器。堆垛机控制系统结构图如图7所示。

\section{2. 系统功能要求}

堆垛机主要动作包括: 水平方向上的行走、载货轿厢 的垂直方向升降、货叉的左右方向伸缩, 所以其整个动作 轨迹可以建立在一个三维坐标系之上, 如图8 所示, 水平 方向上的行走轨迹看作 X 轴, 载货轿厢的垂直方向升降 轨迹看作 Z 轴, 货叉左右方向伸缩轨迹看作 $Y$ 轴。快速 性、平稳性、精准性是堆垛机运行过程中的三个最主要性 能指标，载货或者无货情况都一样。 


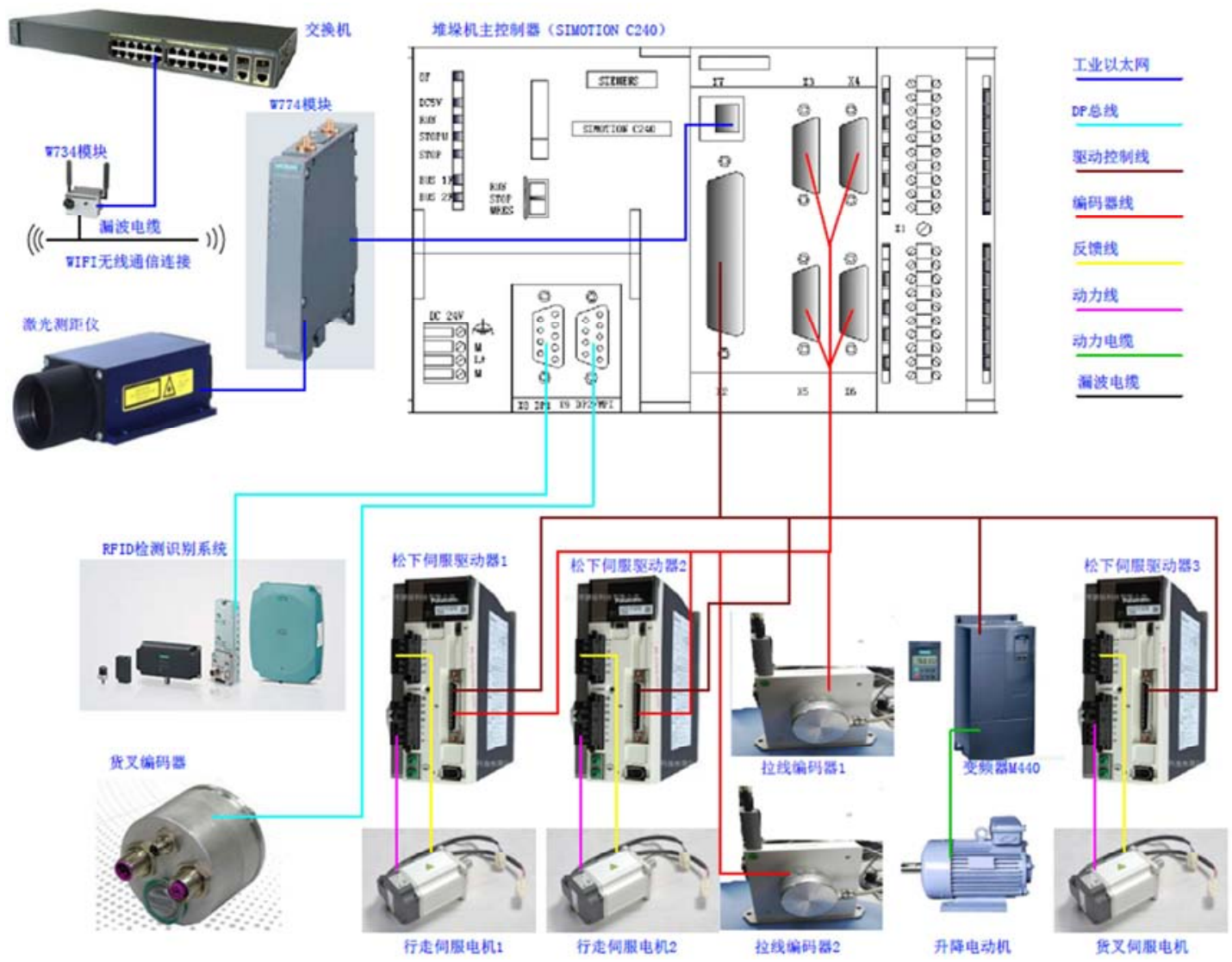

图7 堆垛机控制系统结构图。

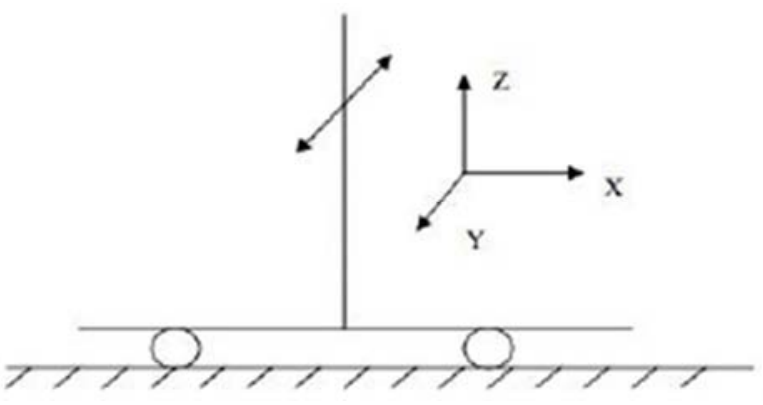

图8 三维坐标系轨迹图。

\subsection{1. 速度要求}

堆垛机运行速度的快慢直接决定着其出、入库的作业 效率，包括水平方向上的行走速度、载货轿厢垂直方向的 升降速度、货叉左右方向的伸缩速度。出、入库作业效率 的提升, 必须依靠三个方向上速度和加速度的提升, 但是 不能速度过大, 速度过大惯性也就越大, 有可能会造成堆 垛机机架的摇晃, 使其机械精度下降, 所以必须找到最优 化的速度控制方案 $[8]$ 。

\subsection{2. 精度要求}

夹具在货架中的存放必须按照统一标准摆放, 不能出 现偏差, 所以取、放夹具时堆垛机就必须有足够高的定位 精度才行, 满足夹具存放的标准化。

\subsection{3. 路径要求}

堆垛机水平方向上的行走和载货轿厢垂直方向上的 升降同步进行, 以更短时间达到目标位置。

\subsection{4. 安全要求}

堆垛机在运行过程中, 必须保证其运行过程的安全, 以免造成不必要的损失。

\section{2 .5 . 货叉要求}

货叉能够准确无误的完成放货 (把夹具存进货位中去) 和取货（从货位中取出夹具）。

\subsection{6. 报警要求}

堆垛机在运行过程中的对其发生的故障必须做到及 时报警并停车，等待操作人员的处理。

\section{3. 堆垛机速度控制方案}

堆垛机的运行从开始到结束可分为加速过程、平稳过 程和减速过程三个过程, 其中加速过程和减速过程的响应 快慢是最关键的, 堆垛机速度变化的性能优劣决定了堆垛 机作业频率的高低, 进而对自动化立体仓库的作业效率有 着很大的影响。另外, 速度控制和位置控制紧密相连, 堆 垛机高速运行中, 快到达目标位置时, 堆垛机要进行减速 制动, 但堆垛机存在运动惯性, 所以, 为了可以精准的停 
在指定货位, 堆垛机必须提早进行减速, 加以缓冲释放惯 性 [9]。堆垛机系统在水平方向、垂直方向、左右方向的 三维立体控制中, 水平运动控制是难点, 因为运行轨迹长 而且运行速度大; 垂直运动相比来说稍简单, 高度低而且 速度小; 货叉左右运动的速度也较低, 且只有高速和低速 两个模式。

传统上实现水平行走的速度控制大部分采用多段曲 线调速（如图9所示）方法来实现水平行走的速度控制。 如果当前仓库有 27 列, 当目标货位距离堆垛机当前位置大 于 9 列时, 采用曲线 1 控制; 大于 3 列小于 9 列时采用曲线 2 控制; 小于 3 列时, 采用曲线 3 控制。

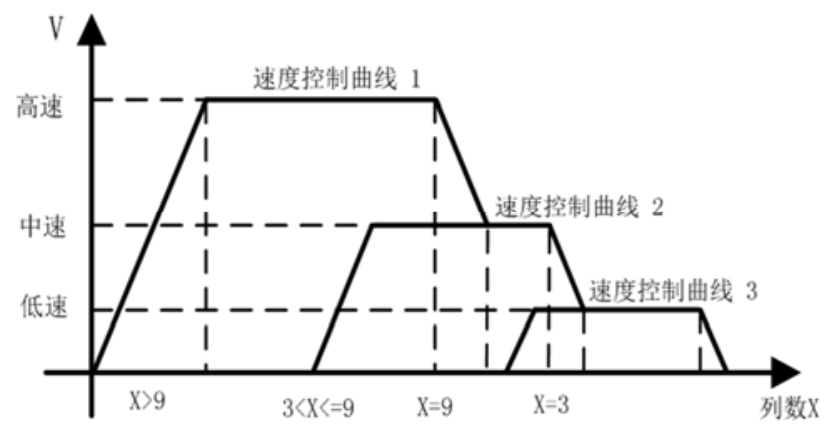

图9 多段曲线调速图。

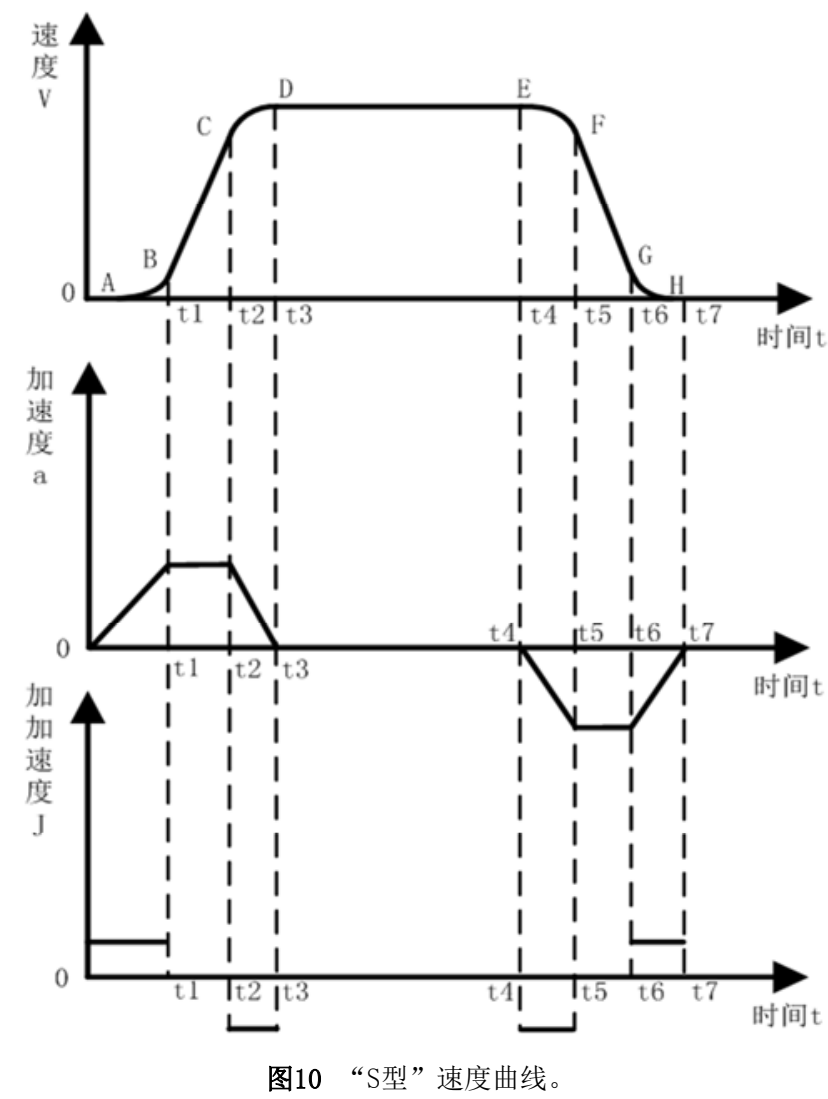

采用此方法由于曲线不是连续的, 加速度的变化是阶 跃的, 启停的瞬间加速度过大, 平稳性得不到保障, 所以 本课题采用 “S型” 曲线 (如图10所示) 调速方法, 此方
法由于曲线连续, 加速度是连续的, 各个动作之间的过渡 更加平滑，使其堆垛机在运行过程中更加的稳定。

其中: $v$ 为速度, $\mathrm{a}$ 为加速度, $\mathrm{J}$ 为加速度变化率。从 图中可以看出 $\mathrm{S}$ 型速度曲线在一个运动周期内包括三个阶 段:

(1) 0 到 $\mathrm{t} 3$ 是加速阶段, 0 到 $\mathrm{t} 1$ 是起动弧线加速段,

$\mathrm{t} 1$ 到 $\mathrm{t} 2$ 加速度固定段, $\mathrm{t} 1$ 到 $\mathrm{t} 2$ 是加速弧线段。

（2）匀速运行阶段： t 3 到 $t 4$ 。

(3) $\mathrm{t} 4$ 的 $\mathrm{t} 7$ 减速阶段, $\mathrm{t} 4$ 到 $\mathrm{t} 5$ 是制动弧线减速段, $\mathrm{t} 5$ 到 $\mathrm{t} 6$ 是减速度固定段, $\mathrm{t} 6$ 到 $\mathrm{t} 7$ 是减速弧线段。

\section{4. 堆垛机定位控制方案}

堆垛机在运行中能否精确位置定位是系统的成功关 键因素, 接收到动作命令后, 通过对目标货位地址的读取, 进行水平方向行走和垂直方向上升、下降动作, 巷道里只 有X方向和Z方向, Y方向为货叉伸缩长度 [10]。

上位调度计算机将命令发送到堆垛机, 堆垛机解析后 自动进行认址。本系统中控制堆垛机水平行走的伺服电机 连接的是增量式伺服反馈编码器, 所以堆垛机长时间工作 会带来一定累积误差, 本系统可以在堆垛机进行作业任务 前使其进行回零运动来消除累积误差, 控制方法简单、可 靠性强 [11]。

\section{5. 主站与子站通信平台的搭建}

为了解决主站PLC 的PN口与子站SIMOTION C240 的 Internet口异构通信的问题, 设计了一个以PLC侧选用 OPEN IE通信方式和SIMOTION侧选用LCom库通信方式的通 信平台, 实现了数据在主站与子站之间稳定、可靠的实时 传输 [12]。通信平台示意图如图11所示。

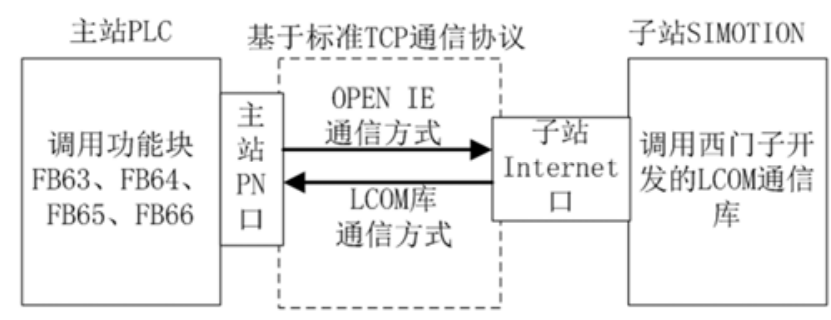

图11 通信平台示意图。

\section{4. 结论}

本文所研究的自动化立体仓库堆垛机采用了 “运动控 制器SIMOTION+伺服驱动器+伺服电机” 的控制结构, 使其 性能得到很大改善, 经过实际运行及调试, 整体运行快速、 平稳、精确。目前, 在进行生产线的焊接夹具的更换, 较 原先采用国内主流的控制结构所设计的自动化立体仓库, 进行生产线的焊接夹具更换, 焊接夹具出入库能力大幅提 高, 使得拆换一副焊接夹具的节拍不大于 $360 \mathrm{~s}$, 其中, 堆 垛机位置控制精度达到 $\pm 0.1 \mathrm{~mm}$, 水平行走速度不低于 $100 \mathrm{~m} / \mathrm{min}$ 、垂直升降速度不低于 $60 \mathrm{~m} / \mathrm{min}$ 、空载货叉速 度不低于 $60 \mathrm{~m} / \mathrm{min}$ 和满载货叉速度不低于 $45 \mathrm{~m} / \mathrm{min}$ 。 


\section{参考文献}

[1] 张欢欢. 自动化立体仓库的若干关键技术与仿真 $[\mathrm{J}]$ : [ 硕士 学位论文]. 杭州: 浙江大学机械与能源工程学院, 2008。

[2] 张建军, 王立波. 自动化立体仓库控制系统设计与实现 [N]. 工业仪表与自动化装置, 2001, 3:36-38。

[3] Byung-In Kim, Robert J, Graves, Sunderesh S. Intelligent agent modeling ofan industrial warehousing problem. IIE Transactions. 2002.

[4] 王涛. 自动化立体仓库堆垛机控制系统的研究 [J] : [硕士 学位论文]. 江西：中北大学控制工程，2015。

[5] Geng, Jingtao and Lu, Yongxia and Yang, Huijing. The design of stereoscopic warehouse stacker' motion and control system [N]. Advances inAutomation and Robotics.

[6] 李小三. 堆垛机控制系统关键技术研究与设计 $[J]$ : [ 硕士学 位论文]. 兰州: 兰州交通大学机械电子工程, 2014。

[7] M. Punnniyamoorth, Ragavan. Justification of automatic storage and retrievalsystem in a heavy engineering industry $[\mathrm{N}]$. The International Journal of AdvancedManufacturing Technology. 2005.

[8] 王勇军, 周奇才. 自动化仓库堆垛机高速运行控制技术 $[N]$. 起重运输机械. $2003,(1): 27^{\sim} 29$ 。

[9] 冯占营, 郭向亮. 自动化立体仓库堆垛机优化控制技术 [N]. 自动控制，2006 (1)：102-103。

[10] D. Wang , C. Li. Self-Adaptive Dynamic Matrix Control for High-Speed Machining Servo Control [J]. The International Journal of Advanced Manufacturing Technology, 2013, 21 (10), 733-738.

[11] 冯勇军. 立体仓库用堆垛机的设计与选用 $[\mathrm{N}]$. 制造业自动 化, 2013, 24:77-79。

[12] 王光东, 朱武, 张佳民. 汽车柔性生产线中立体仓库控制 系统通信平台的设计 $[\mathrm{N}]$ 。仪表技术，2017, 1:17-19。 